\title{
Covariant mass and geometrical setup in Euclidean gauge theories
}

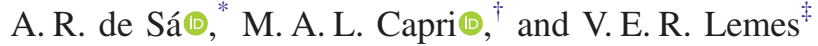 \\ Instituto de Física, Universidade do Estado do Rio de Janeiro, Rua São Francisco Xavier 524, Maracanã, \\ Rio de Janeiro-RJ, 20550-013, Brazil
}

(Received 20 February 2020; accepted 17 April 2020; published 4 May 2020)

\begin{abstract}
A nonlocal mass operator is consistently defined in the local form through the introduction of a set of additional fields with geometrical appropriated properties. A local and polynomial gauge-invariant action is thus established. Equations compatible with the study of renormalization, from the algebraic point of view, are presented in the Landau gauge.
\end{abstract}

DOI: 10.1103/PhysRevD.101.105003

\section{INTRODUCTION}

Nonmaximal dimension condensates are of fundamental interest in order to study the infrared into Euclidean YangMills theories as we can see by the number of results obtained through theoretical, phenomenological, and lattice simulations [1-26]. One particularly important case is the dimension two operator $A_{\mu}^{a} A_{\mu}^{a}$ which is not gauge invariant but is multiplicatively renormalizable to all orders in the Landau gauge. Other dimension two operators can be renormalizable at a large number of other gauges like linear covariant gauges [27], Curci-Ferrari, and the maximal Abelian gauge [26,28].

The fundamental problem of these operators like $A_{\mu}^{a} A_{\mu}^{a}$ and the condensate $\left\langle A_{\mu}^{a} A_{\mu}^{a}\right\rangle$ is that they are gauge dependent and all of their quantum properties must be defined in each gauge. Many attempts in order to present a gauge-invariant mechanism for these operators can be done by [29-31], but the result is always nonunitary, nonrenormalizable, or both. In this way, it is a quite natural objective to discuss and present a suitable colorless dimension two operator $\mathcal{O}(A)$ which preserves gauge invariance,

$$
\begin{aligned}
\delta \mathcal{O}(A) & =0, \\
\delta A_{\mu}^{a} & =-D_{\mu}^{a b} \omega^{b},
\end{aligned}
$$

where $D_{\mu}^{a b}$ is the covariant derivative, given by

\footnotetext{
*alineribeirodesa@gmail.com.br †marcio@dft.if.uerj.br

*verlemes@gmail.com.br
}

Published by the American Physical Society under the terms of the Creative Commons Attribution 4.0 International license. Further distribution of this work must maintain attribution to the author(s) and the published article's title, journal citation, and DOI. Funded by SCOAP ${ }^{3}$.

$$
D_{\mu}^{a b}=\delta^{a b} \partial_{\mu}-g f^{a b c} A_{\mu}^{c} .
$$

The natural candidate for this is the nonlocal operator

$$
\mathcal{O}(A)=-\frac{1}{2} \int d^{4} x F_{\mu \nu}^{a}\left[\left(D^{2}\right)^{-1}\right]^{a b} F_{\mu \nu}^{b}
$$

This operator is studied in [32]. The method developed consists of writing the nonlocal operator into a local form as

$$
\begin{aligned}
& \frac{m^{2}}{4} \int d^{4} x F_{\mu \nu}^{a}\left(\frac{1}{D^{2}}\right)^{a b} F_{\mu \nu}^{b} \\
& \quad \Rightarrow \int d^{4} x\left(\frac{1}{4} \bar{B}_{\mu \nu}^{a} D_{\sigma}^{a b} D_{\sigma}^{b c} B_{\mu \nu}^{c}+\frac{i m}{4}(B-\bar{B})_{\mu \nu}^{a} F_{\mu \nu}^{a}\right)
\end{aligned}
$$

Unfortunately, by means of algebraic renormalization methods, it is possible to observe that a mass counterterm in the tensorial fields can be obtained and that this counterterm turns localization of the gauge-invariant nonlocal mass into an impossibility only by usual antisymmetric tensor fields[32]. In fact, the obtained result implies that it is necessary to include to the quantum action, terms like

$$
\begin{aligned}
- & \frac{3}{8} m^{2} \lambda_{1}\left(\bar{B}_{\mu \nu}^{a} B_{\mu \nu}^{a}\right)+m^{2} \frac{\lambda_{3}}{32}\left(\bar{B}_{\mu \nu}^{a}-B_{\mu \nu}^{a}\right)^{2} \\
& +\frac{\lambda^{a b c d}}{16}\left(\bar{B}_{\mu \nu}^{a} B_{\mu \nu}^{b}\right)\left(\bar{B}_{\rho \sigma}^{c} B_{\rho \sigma}^{d}\right) .
\end{aligned}
$$

These terms make in turn impossible the localization of the gauge-invariant nonlocal mass operator with this simple mechanism. It is important to emphasize here that this analysis in no way prohibits a more elaborate mechanism from achieving this goal. Now, we will present a mechanism that could make it possible to localize the nonlocal operator (3) without the possibility of mass terms in the localizing action that can destroy the process. 


\section{LOCALIZATION OF THE OPERATOR $\int d^{4} x F_{\mu \nu} \frac{1}{D^{2}} F_{\mu \nu}$ WITH SELF-DUAL AND ANTI-SELF-DUAL TENSOR FIELDS}

First, it is necessary to provide a geometrical mechanism that could avoid mass terms in localizing tensorial fields. This geometrical mechanism is recognizable in the self-dual and anti-self-dual properties. In simple terms, two antisymmetric tensor fields that obey the relation $\bar{\varphi}_{\mu \nu} \varphi^{\mu \nu}=0$ are necessary in order to avoid mass term in the localizing fields. The most simple way in order to achieve this goal is to introduce these fields in the localizing action with projectors that have the desired properties, i.e.,

$$
\begin{aligned}
\bar{\theta}_{\mu \nu \alpha \beta} & =\frac{1}{4}\left(\delta_{\mu \alpha} \delta_{\nu \beta}-\delta_{\mu \beta} \delta_{\nu \alpha}+\epsilon_{\mu \nu \alpha \beta}\right) \\
\theta_{\mu \nu \alpha \beta} & =\frac{1}{4}\left(\delta_{\mu \alpha} \delta_{\nu \beta}-\delta_{\mu \beta} \delta_{\nu \alpha}-\epsilon_{\mu \nu \alpha \beta}\right) \\
\bar{\theta}_{\mu \nu \alpha \beta} \bar{\theta}^{\alpha \beta \sigma \lambda} & =\bar{\theta}_{\mu \nu}^{\sigma \lambda} \\
\theta_{\mu \nu \alpha \beta} \theta^{\alpha \beta \sigma \lambda} & =\theta_{\mu \nu}^{\sigma \lambda} \\
\bar{\theta}_{\mu \nu \alpha \beta} \theta^{\alpha \beta \sigma \lambda} & =0 \\
\epsilon_{\alpha \beta \mu \nu} \epsilon^{\alpha \beta \sigma \lambda} & =2 \delta_{[\mu \nu]}^{\sigma \lambda} \\
\delta_{[\mu \nu]}^{\sigma \lambda} & =\delta_{\mu}^{\sigma} \delta_{\nu}^{\lambda}-\delta_{\nu}^{\sigma} \delta_{\mu}^{\lambda} \\
\epsilon_{\alpha \beta \mu \nu} \epsilon^{\alpha \epsilon \sigma \lambda} & =\delta_{\beta}^{\epsilon} \delta_{[\mu \nu]}^{\sigma \lambda}+\delta_{\beta}^{\lambda} \delta_{[\mu \nu]}^{\epsilon \sigma}+\delta_{\beta}^{\sigma} \delta_{[\mu \nu]}^{\lambda \epsilon}
\end{aligned}
$$

and

$$
\begin{aligned}
\bar{\varphi}_{\mu \nu} & =\bar{\theta}_{\mu \nu \alpha \beta} \bar{\varphi}^{\alpha \beta} \\
\varphi_{\mu \nu} & =\theta_{\mu \nu \alpha \beta} \varphi^{\alpha \beta},
\end{aligned}
$$

which lead to the following solution:

$$
\begin{aligned}
\bar{\varphi}_{\mu \nu}=\bar{T}_{\mu \nu}+\tilde{\bar{T}}_{\mu \nu}, & \tilde{\bar{T}}_{\mu \nu}=\frac{1}{2} \epsilon_{\alpha \beta \mu \nu} \bar{T}^{\alpha \beta} \\
\varphi_{\mu \nu}=T_{\mu \nu}-\tilde{T}_{\mu \nu}, & \tilde{T}_{\mu \nu}=\frac{1}{2} \epsilon_{\alpha \beta \mu \nu} T^{\alpha \beta} .
\end{aligned}
$$

This solution, in spite of being interesting, is not the best form to write the action in order to explicitly obtain the set of equations compatible with the quantum action principle. Due to this, we will continue with the fields $\bar{\varphi}_{\mu \nu}$ and $\varphi_{\mu \nu}$. It is important to stress the following property:

$$
\bar{\theta}_{\mu \nu \alpha \beta} \theta^{\mu \gamma \lambda \rho} \neq 0 .
$$

This property is fundamental for the construction of a dynamical kinetic term. It is also important to note that these two properties are also relevant in the study of infrared properties. The generation of mass for all components of the gauge field is different from the usual symmetry breaking. This apparent paradox is solved in this model in a different way as to the one presented in [32]. To understand the mechanism that we will present, it is instructive to remember the original mechanism presented in [32]. It starts adding the nonlocal mass operator to the Yang-Mills action, i.e., considering

$$
S_{\mathrm{YM}}+S_{\mathcal{O}},
$$

where

$$
S_{\mathrm{YM}}=\frac{1}{4} \int d^{4} x F_{\mu \nu}^{a} F_{\mu \nu}^{a}
$$

and

$$
S_{\mathcal{O}}=-\frac{m^{2}}{4} \int d^{4} x F_{\mu \nu}^{a}\left[\left(D^{2}\right)^{-1}\right]^{a b} F_{\mu \nu}^{b} .
$$

The term (12) is localized by means of the introduction of a pair of bosonic antisymmetric tensor fields in the adjoint representation, $\left(B_{\mu \nu}^{a}, \bar{B}_{\mu \nu}^{a}\right)$, according to

$$
\begin{aligned}
e^{-S_{\mathcal{O}}} & =\int D \bar{B} D B\left(\operatorname{det} D^{2}\right)^{6}\left[-S_{\mathcal{O} L}\right] \\
S_{\mathcal{O} L} & \left.=\left(\frac{1}{4} \int d^{4} x \bar{B}_{\mu \nu}^{a} D_{\sigma}^{a b} D_{\sigma}^{b c} B_{\mu \nu}^{c}+\frac{i m}{4} \int d^{4} x(B-\bar{B})_{\mu \nu}^{a} F_{\mu \nu}^{a}\right)\right),
\end{aligned}
$$

where the determinant, $\left(\operatorname{det} D^{2}\right)^{6}$, takes into account the Jacobian arising from the integration over the bosonic fields $\left(\bar{B}_{\mu \nu}^{a}, B_{\mu \nu}^{a}\right)$. This term can also be localized by means of suitable anticommuting antisymmetric tensor fields $\left(\bar{G}_{\mu \nu}^{a}, G_{\mu \nu}^{a}\right)$, namely,

$$
\left(\operatorname{det} D^{2}\right)^{6}=\int D \bar{G} D G \exp \left(\frac{1}{4} \int d^{4} x \bar{G}_{\mu \nu}^{a} D_{\sigma}^{a b} D_{\sigma}^{b c} G_{\mu \nu}^{c}\right) .
$$

The bosonic fields $\left(\bar{B}_{\mu \nu}^{a}, B_{\mu \nu}^{a}\right)$ and the anticommuting fields $\left(\bar{G}_{\mu \nu}^{a}, G_{\mu \nu}^{a}\right)$ form a quartet [32]. Taking into account that the gauge parameter $\omega$ will be promoted to a ghost $c$, this quartet will become a Becchi-Rouet-Stora-Tyutin (BRST) quartet. The complete set of BRST equations for the localizing fields is given by

$$
\begin{aligned}
\delta A_{\mu}^{a} & =-D_{\mu}^{a b} \omega^{b}, \\
\delta B_{\mu \nu}^{a} & =g f^{a b c} \omega^{b} B_{\mu \nu}^{c}+G_{\mu \nu}^{a}, \\
\delta \bar{B}_{\mu \nu}^{a} & =g f^{a b c} \omega^{b} \bar{B}_{\mu \nu}^{c}, \\
\delta G_{\mu \nu}^{a} & =g f^{a b c} \omega^{b} G_{\mu \nu}^{c}, \\
\delta \bar{G}_{\mu \nu}^{a} & =g f^{a b c} \omega^{b} \bar{G}_{\mu \nu}^{c}+\bar{B}_{\mu \nu}^{a},
\end{aligned}
$$

so the kinetic part of the localizing action

$S_{B G}=\frac{1}{4} \int d^{4} x\left(\bar{B}_{\mu \nu}^{a} D_{\sigma}^{a b} D_{\sigma}^{b c} B_{\mu \nu}^{c}-\bar{G}_{\mu \nu}^{a} D_{\sigma}^{a b} D_{\sigma}^{b c} G_{\mu \nu}^{c}\right)$

is left invariant, but the massive part of the localizing action 


$$
S_{m}=\frac{i m}{4} \int d^{4} x(B-\bar{B})_{\mu \nu}^{a} F_{\mu \nu}^{a}
$$

is not invariant. In order to avoid that problem, the mass action $S_{m}$ is replaced by

$$
\frac{1}{4} \int d^{4} x\left(V_{\sigma \rho \mu \nu} \bar{B}_{\sigma \rho}^{a} F_{\mu \nu}^{a}-\bar{V}_{\sigma \rho \mu \nu} B_{\sigma \rho}^{a} F_{\mu \nu}^{a}\right)
$$

At the end, the sources $V_{\sigma \rho \mu \nu}(x), \bar{V}_{\sigma \rho \mu \nu}(x)$ are required to attain their physical value, namely,

$$
\left.\bar{V}_{\sigma \rho \mu \nu}\right|_{\mathrm{phys}}=\left.V_{\sigma \rho \mu \nu}\right|_{\mathrm{phys}}=\frac{-i m}{2}\left(\delta_{\sigma \mu} \delta_{\rho \nu}-\delta_{\sigma \nu} \delta_{\rho \mu}\right),
$$

so that expression (18) gives back the term $S_{m}$. This is essentially a trick in order to treat a noninvariant action term in an algebraic renormalization scheme. Here, it is necessary to emphasize that this mechanism has many problems from the renormalization point of view. In the algebraic renormalization scheme, classical sources can be set to any classical value at any point in the renormalization calculus. We will abandon this mechanism in order to remain strictly into the algebraic renormalization scheme observing that all equations and symmetry identities are compatible with the quantum action principle, i.e., Ward identities with and without linear breaking and Slavnov equation without breaking. There are three points that still need some attention in this procedure. First, we need a geometrical mechanism that can block mass terms like

$$
\tilde{S}_{m}=\int d^{4} x\left[m^{2}\left(\bar{B}_{\mu \nu}^{a} B_{\mu \nu}^{a}-\bar{G}_{\mu \nu}^{a} G_{\mu \nu}^{a}\right)\right]
$$

This can be done by introducing tensor fields coupled to the projectors or obeying the equations as presented in (7). Again, it is important to remember that due to (6) a mass term like the above one is forbidden if the fields $\left(\bar{B}_{\mu \nu}^{a}, B_{\mu \nu}^{a}\right)$ are replaced by $\left(\bar{\theta}_{\mu \nu \alpha \beta} \bar{\varphi}^{a \alpha \beta}, \theta_{\mu \nu \alpha \beta} \varphi^{a \alpha \beta}\right)$. It is also clear that anticommunting fields $\left(\bar{G}_{\mu \nu}^{a}, G_{\mu \nu}^{a}\right)$ are also substituted by $\left(\bar{\theta}_{\mu \nu \alpha \beta} \bar{\omega}^{a \alpha \beta}, \theta_{\mu \nu \alpha \beta} \omega^{a \alpha \beta}\right)$. Second, a quantum mechanism that guarantees that a source be a well-defined classical term and not a specific fixed mass value, ${ }^{1}$ and the third and final point is a way to define two different phases, a massive and a nonmassive one. In order to solve that problem, we will introduce another quartet of scalar fields

\footnotetext{
${ }^{1}$ In order to use algebraic renormalization scheme to ensure the renormalizability of the quantum action, it is necessary that all the transformations for a classical source give rise to another classical source[33]. This only opens the possibility for a symmetry breaking mechanism or a linear soft breaking symmetry.
}

$$
\begin{aligned}
\delta \bar{\psi} & =\bar{\phi} \\
\delta \bar{\phi} & =0 \\
\delta \phi & =\psi \\
\delta \psi & =0
\end{aligned}
$$

and do a symmetry breaking into these scalar fields $(\bar{\phi}, \phi)$. This mechanism is clearly not exactly a standard one. The fundamental difference is that the scalar fields are not linked to a non-Abelian group and due to this, the spontaneous symmetry breaking mechanism gives mass to all components of the non-Abelian gauge field $A_{\mu}^{a}$. Also, the two phases are defined in the symmetry breaking mechanism.

The proper action in order to do that is

$$
\begin{aligned}
S_{\mathrm{YM}+\mathrm{OP}}= & \int d^{4} x\left\{\frac{1}{4} F_{\mu \nu}^{a} F_{\mu \nu}^{a}+\bar{\theta}_{\mu \nu \alpha \beta}\left(D^{\nu} \bar{\varphi}^{\alpha \beta}\right)^{a} \theta^{\mu \sigma \lambda \rho}\left(D_{\sigma} \varphi_{\lambda \rho}\right)^{a}\right. \\
& -\bar{\theta}_{\mu \nu \alpha \beta}\left(D^{\nu} \bar{\omega}^{\alpha \beta}\right)^{a} \theta^{\mu \sigma \lambda \rho}\left(D_{\sigma} \omega_{\lambda \rho}\right)^{a}-i \bar{\phi} \varphi_{\mu \nu}^{a} \theta^{\mu \nu \alpha \beta} F_{\alpha \beta}^{a} \\
& +i \phi \bar{\varphi}_{\mu \nu}^{a} \bar{\theta}^{\mu \nu \alpha \beta} F_{\alpha \beta}^{a}+i \bar{\psi} \omega_{\mu \nu}^{a} \theta^{\mu \nu \alpha \beta} F_{\alpha \beta}^{a} \\
& +i \psi \bar{\omega}_{\mu \nu}^{a} \bar{\theta}^{\mu \nu \alpha \beta} F_{\alpha \beta}^{a}+\partial_{\mu} \bar{\phi} \partial^{\mu} \phi-\partial_{\mu} \bar{\psi} \partial^{\mu} \psi \\
& \left.-m^{2}(\bar{\phi} \phi-\bar{\psi} \psi)+\frac{\lambda}{2}(\bar{\phi} \phi-\bar{\psi} \psi)^{2}\right\}
\end{aligned}
$$

to which adding the Landau gauge fixing action

$$
S_{g f}=\int d^{4} x\left\{i b^{a} \partial^{\mu} A_{\mu}^{a}+\bar{c}^{a} \partial^{\mu}\left(D_{\mu} c\right)^{a}\right\}
$$

determines the action

$$
S=S_{\mathrm{YM}+\mathrm{OP}}+S_{g f},
$$

which is left invariant under the following set of BRST transformations:

$$
\begin{aligned}
s A_{\mu}^{a} & =-\left(\partial_{\mu} c^{a}+g f^{a b c} A_{\mu}^{b} c^{c}\right) \\
s c^{a} & =\frac{g}{2} f^{a b c} c^{b} c^{c} \\
s \bar{\omega}_{\mu \nu}^{a} & =\bar{\varphi}_{\mu \nu}^{a}+g f^{a b c} c^{b} \bar{\omega}_{\mu \nu}^{c} \\
s \bar{\varphi}_{\mu \nu}^{a} & =g f^{a b c} c^{b} \bar{\varphi}_{\mu \nu}^{c} \\
s \varphi_{\mu \nu}^{a} & =\omega_{\mu \nu}^{a}+g f^{a b c} c^{b} \varphi_{\mu \nu}^{c} \\
s \omega_{\mu \nu}^{a} & =g f^{a b c} c^{b} \omega_{\mu \nu}^{c} \\
s \bar{\psi} & =\bar{\phi} \\
s \bar{\phi} & =0 \\
s \phi & =\psi \\
s \psi & =0 .
\end{aligned}
$$

It is now necessary to say a few words about the scalar sector and spontaneous symmetry breaking. 
The action (22) is invariant under a global $U(1)$ transformation which is

$$
\begin{aligned}
\bar{\phi} & \rightarrow e^{i \Lambda} \bar{\phi} \quad \bar{\varphi}_{\mu \nu}^{a} \rightarrow e^{-i \Lambda} \bar{\varphi}_{\mu \nu}^{a} \\
\phi & \rightarrow e^{-i \Lambda} \phi \quad \varphi_{\mu \nu}^{a} \rightarrow e^{i \Lambda} \varphi_{\mu \nu}^{a} \\
\bar{\psi} & \rightarrow e^{i \Lambda} \bar{\psi} \quad \bar{\omega}_{\mu \nu}^{a} \rightarrow e^{-i \Lambda} \bar{\omega}_{\mu \nu}^{a} \\
\psi & \rightarrow e^{-i \Lambda} \psi \quad \omega_{\mu \nu}^{a} \rightarrow e^{i \Lambda} \omega_{\mu \nu}^{a},
\end{aligned}
$$

and these symmetries, together with (25), are enough to ensure that

$$
\begin{aligned}
V(\bar{\phi}, \phi ; \bar{\psi}, \psi) & =V(\bar{\phi} \phi ; \bar{\psi} \psi) \\
V(\bar{\phi} \phi ; \bar{\psi} \psi) & =V(\bar{\phi} \phi-\bar{\psi} \psi) .
\end{aligned}
$$

Looking to (22), the potential

$$
V(\bar{\phi} \phi-\bar{\psi} \psi)=-m^{2}(\bar{\phi} \phi-\bar{\psi} \psi)+\frac{\lambda}{2}(\bar{\phi} \phi-\bar{\psi} \psi)^{2}
$$

admits a nonzero expectation value for the scalar field

$$
\frac{\partial V}{\partial \phi}=0 \Rightarrow\langle\bar{\phi} \phi\rangle=\frac{m^{2}}{\lambda} .
$$

Due to the $U(1)$ global symmetry, it is necessary to take

$$
\langle\bar{\phi}\rangle=\langle\phi\rangle=\frac{m}{\sqrt{\lambda}}=\mu .
$$

It is important to emphasize here that in order to obtain localization $\langle\bar{\phi}\rangle$ and $\langle\phi\rangle$ always appear in the propagator and observables as a pair $\langle\bar{\phi}\rangle\langle\phi\rangle$. The redefinitions $\bar{\phi} \rightarrow$ $\bar{\phi}-\langle\bar{\phi}\rangle$ and $\phi \rightarrow \phi-\langle\phi\rangle$ are the only necessary requirement in order to obtain localization, and after the integration over the localizing fields, the bilinear term is given by

$$
S_{\text {mass }}=\int d^{4} x\left\{4\langle\bar{\phi}\rangle\langle\phi\rangle A_{\mu}^{a}\left(\delta^{\mu \nu}-\frac{\partial^{\mu} \partial^{\nu}}{\partial^{2}}\right) A_{\nu}^{a}\right\},
$$

which is the desired mass contribution for the free propagator. In a future section, we will discuss in detail the gauge propagator. It is also important that is possible to use such mechanism to study certain topologically nontrivial solutions to the gauge field.

\section{A. Small comment about the symmetry breaking mechanism}

It is clear that the action (22) is constructed in the form

$$
S_{\mathrm{YM}+\mathrm{OP}}=\int d^{4} x\left\{\frac{1}{4} F_{\mu \nu}^{a} F_{\mu \nu}^{a}+s(\Theta)\right\},
$$

where $\Theta$ is constructed with ultraviolet dimension 4 and ghost number -1 with the localizing and gauge fields. In the symmetric phase, $s(\Theta)$ is a trivial term and the cohomology property ensures that the action is pure YangMills from the geometrical point of view. The situation in the broken phase is a little different. Let us look at the terms of interaction of the localizing fields and the curvature. They are obtained as

$$
\begin{aligned}
\Theta= & \bar{\theta}_{\mu \nu \alpha \beta}\left(D^{\nu} \bar{\omega}^{\alpha \beta}\right)^{a} \theta^{\mu \sigma \lambda \rho}\left(D_{\sigma} \varphi_{\lambda \rho}\right)^{a} \\
& -i \bar{\psi} \varphi_{\mu \nu}^{a} \theta^{\mu \nu \alpha \beta} F_{\alpha \beta}^{a}+i \phi \bar{\omega}_{\mu \nu}^{a} \bar{\theta}^{\mu \nu \alpha \beta} F_{\alpha \beta}^{a} \\
s(\Theta)= & \bar{\theta}_{\mu \nu \alpha \beta}\left(D^{\nu} \bar{\varphi}^{\alpha \beta}\right)^{a} \theta^{\mu \sigma \lambda \rho}\left(D_{\sigma} \varphi_{\lambda \rho}\right)^{a} \\
& -\bar{\theta}_{\mu \nu \alpha \beta}\left(D^{\nu} \bar{\omega}^{\alpha \beta}\right)^{a} \theta^{\mu \sigma \lambda \rho}\left(D_{\sigma} \omega_{\lambda \rho}\right)^{a} \\
& -i \bar{\phi} \varphi_{\mu \nu}^{a} \theta^{\mu \nu \alpha \beta} F_{\alpha \beta}^{a}+i \phi \bar{\varphi}_{\mu \nu}^{a} \bar{\theta}^{\mu \nu \alpha \beta} F_{\alpha \beta}^{a} \\
& +i \bar{\psi} \omega_{\mu \nu}^{a} \theta^{\mu \nu \alpha \beta} F_{\alpha \beta}^{a}+i \psi \bar{\omega}_{\mu \nu}^{a} \bar{\theta}^{\mu \nu \alpha \beta} F_{\alpha \beta}^{a} .
\end{aligned}
$$

In the broken phase, the set of BRST symmetries for the scalar fields become

$$
\begin{aligned}
s \bar{\psi} & =\bar{\phi}-\mu \\
s \bar{\phi} & =0 \\
s \phi & =\psi \\
s \psi & =0,
\end{aligned}
$$

where $\mu$ is defined in (30). Applying now the same procedure to the localizing sector above, now we obtain one more term $i \mu \varphi_{\mu \nu}^{a} \theta^{\mu \nu \alpha \beta} F_{\alpha \beta}^{a}$. It is clear that by doing the shift $\phi \Rightarrow \phi-\mu$ and $\bar{\phi} \Rightarrow \bar{\phi}-\mu$ into the action, another term is obtained. The term is $-i \mu \bar{\varphi}_{\mu \nu}^{a} \bar{\theta}^{\mu \nu \alpha \beta} F_{\alpha \beta}^{a}$ which is gauge invariant. The relation of this term to other terms of this sector is provided by a symmetry,

$\int d^{4} x\left\{\bar{\varphi}_{\mu \nu}^{a} \frac{\delta S}{\delta \bar{\varphi}_{\mu \nu}^{a}}-\varphi_{\mu \nu}^{a} \frac{\delta S}{\delta \varphi_{\mu \nu}^{a}}+\bar{\phi} \frac{\delta S}{\delta \bar{\phi}}-\phi \frac{\delta S}{\delta \phi}\right\}=0$,

which can be easily extended to the broken phase and means that the U(1) symmetry presented in (26) is broken,

$$
\begin{aligned}
& \int d^{4} x\left\{\bar{\varphi}_{\mu \nu}^{a} \frac{\delta S_{\mu}}{\delta \bar{\varphi}_{\mu \nu}^{a}}-\varphi_{\mu \nu}^{a} \frac{\delta S_{\mu}}{\delta \varphi_{\mu \nu}^{a}}+(\bar{\phi}-\mu) \frac{\delta S_{\mu}}{\delta \bar{\phi}}-(\phi-\mu) \frac{\delta S_{\mu}}{\delta \phi}\right\}=0 \\
& S_{\mu}=S(\langle\varphi\rangle=\mu ;\langle\bar{\varphi}\rangle=\mu) .
\end{aligned}
$$

This equation does the link between the two phases and fixes the value of the mass in a similar way as used in the symmetry breaking mechanism. The main difference between this and the usual one is that mass for all the $A_{\nu}^{a}$ which is also the same that appears in DelbourgoThompson [29-31] mechanism is avoided here. 


\section{EXTENDING TO THE QUANTUM LEVEL}

The set of equations, valid at quantum level as any equation that obeys the quantum action principle (QAP) [33], that ensure that no mixing term of the form $\bar{\varphi}_{\mu \nu}^{a} \varphi_{\mu \nu}^{a}$ is permitted are

$$
\begin{aligned}
& \theta_{\mu \nu \alpha \beta} \frac{\delta S}{\delta \bar{\varphi}_{\alpha \beta}^{a}}=0 \\
& \bar{\theta}_{\mu \nu \alpha \beta} \frac{\delta S}{\delta \varphi_{\alpha \beta}^{a}}=0 \\
& \theta_{\mu \nu \alpha \beta} \frac{\delta S}{\delta \bar{\omega}_{\alpha \beta}^{a}}=0 \\
& \bar{\theta}_{\mu \nu \alpha \beta} \frac{\delta S}{\delta \omega_{\alpha \beta}^{a}}=0 .
\end{aligned}
$$

In order to present all equations compatible with the quantum action principle, it is necessary to add to the action all the symmetries coupled to classical sources. It is interesting to remember here that due to the properties of the projectors it is useful to introduce the projectors explicitly into the source terms in a way that the projections over the source equations are also obtained. It is clear that introducing directly the projectors with the sources the functional derivative in respect to the sources does not give us only the symmetry associated to these source but instead we obtain the projected symmetry which is also a symmetry of the action due to the operator idempotency. The Landau gauge fixing action plus the symmetries is given by

$$
\begin{aligned}
S_{J}= & \int d^{4} x\left\{-\Omega^{a}\left(D_{\mu} C\right)^{a}\right. \\
& +L^{a} \frac{g}{2} f^{a b c} c^{b} c^{c}+\bar{J}_{\alpha \beta}^{a} \theta^{\alpha \beta \mu \nu}\left(\omega_{\mu \nu}^{a}+g f^{a b c} c^{b} \varphi_{\mu \nu}^{c}\right) \\
& +J_{\alpha \beta}^{a} \bar{\theta}^{\alpha \beta \mu \nu}\left(g f^{a b c} c^{b} \bar{\varphi}_{\mu \nu}^{c}\right)+\chi_{\alpha \beta}^{a} \bar{\theta}^{\alpha \beta \mu \nu}\left(\bar{\varphi}_{\mu \nu}^{a}+g f^{a b c} c^{b} \bar{\omega}_{\mu \nu}^{c}\right) \\
& \left.+\bar{\chi}_{\alpha \beta}^{a} \theta^{\alpha \beta \mu \nu}\left(g f^{a b c} c^{b} \omega_{\mu \nu}^{c}\right)\right\},
\end{aligned}
$$

which has over the sources the same type of property as presented over the equations of motion for the fields

$$
\begin{aligned}
& \bar{\theta}_{\mu \nu \alpha \beta} \frac{\delta S^{J}}{\delta \bar{J}_{\alpha \beta}^{a}}=0 \\
& \theta_{\mu \nu \alpha \beta} \frac{\delta S^{J}}{\delta J_{\alpha \beta}^{a}}=0 \\
& \bar{\theta}_{\mu \nu \alpha \beta} \frac{\delta S^{J}}{\delta \bar{\chi}_{\alpha \beta}^{a}}=0 \\
& \theta_{\mu \nu \alpha \beta} \frac{\delta S^{J}}{\delta \chi_{\alpha \beta}^{a}}=0 .
\end{aligned}
$$

Now the quantum actions and the Slavnov-Taylor identity are given by

$$
\begin{aligned}
\Sigma= & S+S_{g f}+S_{J} \\
S(\Sigma)= & \int d^{4} x\left\{\frac{\delta \Sigma}{\delta A_{\mu}^{a}} \frac{\delta \Sigma}{\delta \Omega_{\mu}^{a}}+\frac{\delta \Sigma}{\delta c^{a}} \frac{\delta \Sigma}{\delta L^{a}}+\frac{\delta \Sigma}{\delta \bar{\varphi}_{\alpha \beta}^{a}} \frac{\delta \Sigma}{\delta J_{\alpha \beta}^{a}}\right. \\
& +\frac{\delta \Sigma}{\delta \varphi_{\alpha \beta}^{a}} \frac{\delta \Sigma}{\delta \bar{J}_{\alpha \beta}^{a}}+\frac{\delta \Sigma}{\delta \bar{\omega}_{\alpha \beta}^{a}} \frac{\delta \Sigma}{\delta \chi_{\alpha \beta}^{a}}+\frac{\delta \Sigma}{\delta \omega_{\alpha \beta}^{a}} \frac{\delta \Sigma}{\delta \bar{\chi}_{\alpha \beta}^{a}} \\
& \left.+\bar{\phi} \frac{\delta \Sigma}{\delta \bar{\psi}}+\psi \frac{\delta \Sigma}{\delta \phi}+i b^{a} \frac{\delta \Sigma}{\delta \bar{c}^{a}}\right\},
\end{aligned}
$$

and the self-dual and anti-self-dual equations extended to the quantum action are

$$
\begin{aligned}
& \theta_{\mu \nu \alpha \beta} \frac{\delta \Sigma}{\delta \bar{\varphi}_{\alpha \beta}^{a}}=0 \\
& \bar{\theta}_{\mu \nu \alpha \beta} \frac{\delta \Sigma}{\delta \varphi_{\alpha \beta}^{a}}=0 \\
& \theta_{\mu \nu \alpha \beta} \frac{\delta \Sigma}{\delta \bar{\omega}_{\alpha \beta}^{a}}=0 \\
& \bar{\theta}_{\mu \nu \alpha \beta} \frac{\delta \Sigma}{\delta \omega_{\alpha \beta}^{a}}=0 \\
& \bar{\theta}_{\mu \nu \alpha \beta} \frac{\delta \Sigma}{\delta \bar{J}_{\alpha \beta}^{a}}=0 \\
& \theta_{\mu \nu \alpha \beta} \frac{\delta \Sigma}{\delta J_{\alpha \beta}^{a}}=0 \\
& \bar{\theta}_{\mu \nu \alpha \beta} \frac{\delta \Sigma}{\delta \bar{\chi}_{\alpha \beta}^{a}}=0 \\
& \theta_{\mu \nu \alpha \beta} \frac{\delta \Sigma}{\delta \chi_{\alpha \beta}^{a}}=0 .
\end{aligned}
$$

It is important to emphasize again that this set of equations simply block the possibility of mass terms like $\bar{\varphi}_{\alpha \beta}^{a} \varphi^{\alpha \beta a}$ or $\bar{\omega}_{\alpha \beta}^{a} \omega^{\alpha \beta a}$ and thus turn the mass term obtained from the localization of the nonlocal operator presented in action and the symmetry breaking mechanism (22) into a stable one. Also, this set of equations block a quartic term for the localizing fields due to the simple fact that every antisymmetric tensor of rank $D+1$ in $D$ dimensions is zero than it is always possible to rewrite the indexes of a quadratic term in order to be forbidden by this set of equations.

The linearized operator $\beta_{\Sigma}$ is easily obtained from the Slavnov-Taylor identity and is given by 


$$
\begin{aligned}
\beta_{\Sigma}= & \int d^{4} x\left\{\frac{\delta \Sigma}{\delta A_{\mu}^{a}} \frac{\delta}{\delta \Omega_{\mu}^{a}}+\frac{\delta \Sigma}{\delta \Omega_{\mu}^{a}} \frac{\delta}{\delta A_{\mu}^{a i}}\right. \\
& +\frac{\delta \Sigma}{\delta c^{a}} \frac{\delta}{\delta L^{a}}+\frac{\delta \Sigma}{\delta L^{a}} \frac{\delta}{\delta c^{a}}+\frac{\delta \Sigma}{\delta \bar{\varphi}_{\alpha \beta}^{a}} \frac{\delta}{\delta J_{\alpha \beta}^{a}}+\frac{\delta \Sigma}{\delta J_{\alpha \beta}^{a}} \frac{\delta}{\delta \bar{\varphi}_{\alpha \beta}^{a}} \\
& +\frac{\delta \Sigma}{\delta \varphi_{\alpha \beta}^{a}} \frac{\delta}{\delta \bar{J}_{\alpha \beta}^{a}}+\frac{\delta \Sigma}{\delta \bar{J}_{\alpha \beta}^{a}} \frac{\delta}{\delta \varphi_{\alpha \beta}^{a}}+\frac{\delta \Sigma}{\delta \bar{\omega}_{\alpha \beta}^{a}} \frac{\delta}{\delta \chi_{\alpha \beta}^{a}}+\frac{\delta \Sigma}{\delta \chi_{\alpha \beta}^{a}} \frac{\delta}{\delta \bar{\omega}_{\alpha \beta}^{a}} \\
& \left.+\frac{\delta \Sigma}{\delta \omega_{\alpha \beta}^{a}} \frac{\delta}{\delta \bar{\chi}_{\alpha \beta}^{a}}+\frac{\delta \Sigma}{\delta \bar{\chi}_{\alpha \beta}^{a}} \frac{\delta}{\delta \omega_{\alpha \beta}^{a}}+\bar{\phi} \frac{\delta}{\delta \bar{\psi}}+\psi \frac{\delta}{\delta \phi}+i b^{a} \frac{\delta}{\delta \bar{c}^{a}}\right\} .
\end{aligned}
$$

The gauge fixing, the antighost equation, and the ghost equation that are characteristics of the Landau gauge are given by ${ }^{2}$

$$
\begin{aligned}
\frac{\delta \Sigma}{\delta b^{a}} & =i \partial^{\mu} A_{\mu}^{a} \\
\frac{\delta \Sigma}{\delta \bar{c}^{a}}+\partial_{\mu} \frac{\delta \Sigma}{\delta \Omega_{\mu}^{a}} & =0 \\
G^{a}(\Sigma) & =\int d^{4} x\left\{\frac{\delta \Sigma}{\delta c^{a}}+i g f^{a b c} \bar{c}^{b} \frac{\delta \Sigma}{\delta b^{c}}\right\} \\
\Delta^{a} & =\int d^{4} x g f^{a b c}\left\{\Omega_{\mu}^{b i} A^{c \mu}-L^{b} c^{c}+\theta_{\mu \nu \alpha \beta}\left(\bar{J}^{\mu \nu b} \varphi^{\alpha \beta c}-\bar{\chi}^{\mu \nu b} \omega^{\alpha \beta c}\right)+\bar{\theta}_{\mu \nu \alpha \beta}\left(J^{\mu \nu b} \bar{\varphi}^{\alpha \beta c}-\chi^{\mu \nu b} \bar{\omega}^{\alpha \beta c}\right)\right\} \\
G^{a}(\Sigma) & =\Delta^{a} .
\end{aligned}
$$

The rigid equation that corresponds, in the Landau gauge, to the anticommutation of the ghost equation and the Slavnov one is

$$
\begin{aligned}
\left\{G^{a}, \beta_{\Sigma}\right\}= & -W^{a} \\
W^{a i}= & \int d^{4} x g f^{a b c}\left\{A_{\mu}^{b} \frac{\delta}{\delta A_{\mu}^{c}}+\Omega_{\mu}^{b} \frac{\delta}{\delta \Omega_{\mu}^{c}}+L^{b} \frac{\delta}{\delta L^{c}}+c^{b} \frac{\delta}{\delta c^{c}}+b^{b} \frac{\delta}{\delta b^{c}}+\bar{c}^{b} \frac{\delta}{\delta \bar{c}^{c}}\right. \\
& +\theta_{\mu \nu \alpha \beta}\left(\bar{J}^{\mu \nu b} \frac{\delta}{\delta \bar{J}_{\alpha \beta}^{c}}+\bar{\chi}^{\mu \nu b i} \frac{\delta}{\delta \bar{\chi}_{\alpha \beta}^{c}}+\varphi^{\mu \nu b} \frac{\delta}{\delta \varphi_{\alpha \beta}^{c}}+\omega^{\mu \nu b} \frac{\delta}{\delta \omega_{\alpha \beta}^{c i}}\right) \\
& \left.+\bar{\theta}_{\mu \nu \alpha \beta}\left(J^{\mu \nu b i} \frac{\delta}{\delta \bar{J}_{\alpha \beta}^{c}}+\chi^{\mu \nu b} \frac{\delta}{\delta \chi_{\alpha \beta}^{c}}+\bar{\varphi}^{\mu \nu b} \frac{\delta}{\delta \bar{\varphi}_{\alpha \beta}^{c}}+\bar{\omega}^{\mu \nu b} \frac{\delta}{\delta \bar{\omega}_{\alpha \beta}^{c}}\right)\right\} .
\end{aligned}
$$

Also, another set of equations that are compatible with the QAP are

$$
\begin{aligned}
Q(\Sigma) & =\int d^{4} x\left\{\bar{\varphi}_{\mu \nu}^{a} \frac{\delta \Sigma}{\delta \bar{\varphi}_{\mu \nu}^{a}}-\varphi_{\mu \nu}^{a} \frac{\delta \Sigma}{\delta \varphi_{\mu \nu}^{a}}+\bar{J}_{\mu \nu}^{a} \frac{\delta \Sigma}{\delta \bar{J}_{\mu \nu}^{a}}-J_{\mu \nu}^{a} \frac{\delta \Sigma}{\delta J_{\mu \nu}^{a}}+\bar{\phi} \frac{\delta \Sigma}{\delta \bar{\phi}}-\phi \frac{\delta \Sigma}{\delta \phi}\right\} \\
\Delta & =\int d^{4} x\left\{\bar{\theta}^{\mu \nu \alpha \beta} \chi_{\mu \nu}^{a i} \bar{\varphi}_{\alpha \beta}^{a}+\theta^{\mu \nu \alpha \beta} \bar{J}_{\mu \nu}^{a} \chi_{\alpha \beta}^{a}\right\} \\
Q(\Sigma) & =\Delta, \\
R(\Sigma) & =\int d^{4} x\left\{\bar{\omega}_{\mu \nu}^{a} \frac{\delta \Sigma}{\delta \bar{\omega}_{\mu \nu}^{a}}-\omega_{\mu \nu}^{a} \frac{\delta \Sigma}{\delta \omega_{\mu \nu}^{a}}+\bar{\chi}_{\mu \nu}^{a} \frac{\delta \Sigma}{\delta \bar{\chi}_{\mu \nu}^{a}}-\chi_{\mu \nu}^{a} \frac{\delta \Sigma}{\delta \chi_{\mu \nu}^{a}}+\bar{\psi} \frac{\delta \Sigma}{\delta \bar{\psi}}-\psi \frac{\delta \Sigma}{\delta \psi}\right\} \\
R(\Sigma) & =-\Delta .
\end{aligned}
$$

\footnotetext{
${ }^{2}$ These sets of equations ensure that the ghost fields do not renormalize in the Landau gauge.
} 
The sum of Eqs. (45) and (46) corresponds to a local implementation of the $U(1)$ (26) symmetry and to the quantum implementation of (26),

$$
\begin{aligned}
{\left[Q, \beta_{\Sigma}\right] } & =\eta \\
\eta(\Sigma) & =\int d^{4} x\left\{\bar{\phi} \frac{\delta \Sigma}{\delta \bar{\psi}}+\psi \frac{\delta \Sigma}{\delta \phi}+\bar{\theta}^{\mu \nu \alpha \beta}\left(\bar{\varphi}_{\mu \nu}^{a} \frac{\delta \Sigma}{\delta \bar{\omega}_{\alpha \beta}^{a}}-\chi_{\mu \nu}^{a} \frac{\delta \Sigma}{\delta J_{\alpha \beta}^{a}}\right)+\theta^{\mu \nu \alpha \beta}\left(\omega_{\mu \nu}^{a} \frac{\delta \Sigma}{\delta \varphi_{\alpha \beta}^{a}}-\bar{J}_{\mu \nu}^{a} \frac{\delta \Sigma}{\delta \bar{\chi}_{\alpha \beta}^{a}}\right)\right\}
\end{aligned}
$$

This set of equations, with the hermiticity condition, is enough to guarantee that no mass term for the localizing fields exists. Now, it is necessary to guarantee that at the bilinear level the localizing action generates a mass term for the gauge fields $A_{\mu}^{a}$. In order to do that, it is enough to take the bilinear action and integrate over the localizing fields.

\section{A. Bilinear sector of the localizing fields and the propagators}

Taking into account the bilinear part of the gauge functional with localizing fields and the gauge field, it is possible to do the integration in the localizing fields,

$$
\begin{aligned}
Z[A, c, b]= & \int D A_{\mu} \int D \varphi D \bar{\varphi} e^{-S_{o}\left(\bar{\varphi}, \varphi, A_{\mu}\right)} \\
S_{0(\bar{\varphi}, \varphi)}= & \int d^{4} x\left\{\bar{\theta}_{\mu \nu \alpha \beta}\left(\partial^{\nu} \bar{\varphi}^{\alpha \beta}\right)^{a} \theta^{\mu \sigma \lambda \rho}\left(\partial_{\sigma} \varphi_{\lambda \rho}\right)^{a}\right. \\
& \left.-i a \mu \varphi_{\mu \nu}^{a} \theta^{\mu \nu \alpha \beta} F_{0 \alpha \beta}^{a}+i a \mu \bar{\varphi}_{\mu \nu}^{a} \bar{\theta}^{\mu \nu \alpha \beta} F_{0 \alpha \beta}^{a}\right\} \\
F_{0 \alpha \beta}^{a}= & \partial_{\alpha} A_{\beta}^{a}-\partial_{\beta} A_{\alpha}^{a},
\end{aligned}
$$

where $\langle\bar{\phi}\rangle=\mu$ and $\langle\phi\rangle=\mu$ are the vacuum obtained from a spontaneous symmetry breaking mechanism over the fields $\phi$ and $\bar{\phi}$. In order to do that integration, it is enough to obtain the classical equations of motion which are

$$
\begin{aligned}
& -\left\{\bar{\theta}_{\mu \nu \alpha \beta} \theta^{\mu \sigma \lambda \rho}\left(\partial^{\nu} \partial_{\sigma} \varphi_{\lambda \rho}\right)^{a}\right\}+i a \mu \bar{\theta}_{\mu \nu \alpha \beta} F^{0 a \mu \nu}=0, \\
& -\left\{\theta_{\mu \nu \alpha \beta} \bar{\theta}^{\mu \sigma \lambda \rho}\left(\partial^{\nu} \partial_{\sigma} \bar{\varphi}_{\lambda \rho}\right)^{a}\right\}-i a \mu \theta_{\mu \nu \alpha \beta} F^{0 a \mu \nu}=0 .
\end{aligned}
$$

The classical solution for this set of equations of motion is of the form

$$
\begin{aligned}
& \varphi_{\mu \nu}^{a}=4 i a \mu \frac{1}{\partial^{2}} \theta_{\mu \nu \alpha \beta} F_{0}^{\alpha \beta a} \\
& \bar{\varphi}_{\mu \nu}^{a}=-4 i a \mu \frac{1}{\partial^{2}} \bar{\theta}_{\mu \nu \alpha \beta} F_{0}^{\alpha \beta a} .
\end{aligned}
$$

Integrating over the localizing fields, it is easy to find the contribution for the mass of the gauge field as

$$
S_{\mathrm{mass}}=\int d^{4} x\left\{4(a)^{2} \mu^{2} A_{\mu}^{a}\left(\delta^{\mu \nu}-\frac{\partial^{\mu} \partial^{\nu}}{\partial^{2}}\right) A_{\nu}^{a}\right\} .
$$

It is clear that $S_{\text {mass }}$ corresponds to the bilinear contribution to the gauge-invariant mass term presented in (22) for the value $a=1$. It is important to emphasize here that the mass term is proportional to

$$
\int d^{4} x\left\{A_{\mu}^{a T} A^{\mu a T}\right\}
$$

where

$$
A_{\mu}^{a T}=\left(\delta^{\mu \nu}-\frac{\partial^{\mu} \partial^{\nu}}{\partial^{2}}\right) A_{\nu}^{a}
$$

is the transverse part of the gauge field. The complete nonAbelian extension could be understood as the localization of $A_{\min }^{2}$. These operators, defined as

$$
\begin{aligned}
A_{\min }^{2} & \equiv \min _{u} \operatorname{Tr} \int d^{4} x\left\{A_{\mu}^{u} A^{\mu u}\right\} \\
A_{\mu}^{u} & =u^{\dagger} A_{\mu} u+\frac{i}{g} u^{\dagger} \partial_{\mu} u,
\end{aligned}
$$

and their relation to a non-Abelian nonlocal operator have been obtained in Ref. [32].

Taking into account that we are working on the Landau gauge, it is easy to obtain ${ }^{3}$

$$
\left\langle A_{\mu}^{a}(-k) A_{\nu}^{b}(k)\right\rangle=-2 \delta^{a b}\left(\delta^{\mu \nu}-\frac{k^{\mu} k^{\nu}}{k^{2}}\right) \frac{1}{k^{2}+8(a)^{2} \mu^{2}} .
$$

Performing some calculations, we obtain for all the propagators

\footnotetext{
${ }^{3}$ We are using the convention that $\langle\Theta(-k) \Theta(k)\rangle=-G_{\Theta \Theta}$.
} 


$$
\begin{aligned}
\left\langle\bar{\varphi}_{\mu \nu}^{a}(-k) A_{\gamma}^{b}(k)\right\rangle= & -\delta^{a b} \frac{2}{a \mu}\left(\frac{1}{k^{2}}-\frac{1}{k^{2}+8(a)^{2} \mu^{2}}\right) \bar{\theta}_{\mu \nu \gamma \sigma} k^{\sigma} \\
\left\langle\varphi_{\mu \nu}^{a}(-k) A_{\gamma}^{b}(k)\right\rangle= & \delta^{a b} \frac{2}{a \mu}\left(\frac{1}{k^{2}}-\frac{1}{k^{2}+8(a)^{2} \mu^{2}}\right) \theta_{\mu \nu \gamma \sigma} k^{\sigma} \\
\left\langle\varphi_{\sigma \lambda}^{a}(k) \bar{\varphi}_{\mu \nu}^{b}(-k)\right\rangle= & \delta^{a b} \frac{2}{k^{2}+8(a)^{2} \mu^{2}}\left(\theta_{\sigma \lambda \rho \epsilon^{k}} \bar{\theta}_{\mu \nu \alpha \gamma} k^{\gamma} \delta^{\rho \alpha}\right) \\
& \times\left(\frac{1}{k^{2}}\right) \\
\left\langle\omega_{\sigma \lambda}^{a}(k) \bar{\omega}_{\mu \nu}^{b}(-k)\right\rangle & =-\delta^{a b} \frac{2}{k^{2}}\left(\theta_{\sigma \lambda \rho \epsilon} k^{\epsilon} \bar{\theta}_{\mu \nu \alpha \gamma} k^{\gamma} \delta^{\rho \alpha}\right)\left(\frac{1}{k^{2}}\right) \\
\langle\bar{\phi}(-k) \phi(k)\rangle & =-\frac{1}{2}\left(\frac{1}{k^{2}+2 \lambda \mu^{2}}+\frac{1}{k^{2}}\right) \\
\langle\bar{\phi}(-k) \bar{\phi}(k)\rangle & =\langle\phi(-k) \phi(k)\rangle=-\frac{1}{2}\left(\frac{1}{k^{2}+2 \lambda \mu^{2}}-\frac{1}{k^{2}}\right) \\
\langle\bar{\psi}(-k) \psi(k)\rangle & =\frac{1}{k^{2}} \\
\left\langle b^{a}(-k) A_{\nu}^{b}(k)\right\rangle & =-\delta^{a b} \frac{k_{\nu}}{k^{2}} \\
\left\langle\bar{c}^{a}(-k) c^{b}(k)\right\rangle & =\frac{\delta^{a b}}{k^{2}} .
\end{aligned}
$$

This set of propagators confirm our assumption that this mechanism generates a transverse mass term for the gauge field. It is also possible to observe the nonmassive poles in $\left\langle\bar{\varphi}_{\mu \nu}^{a}(-k) A_{\gamma}^{b}(k)\right\rangle$ and $\left\langle\varphi_{\mu \nu}^{a}(-k) A_{\gamma}^{b}(k)\right\rangle$ expected from the symmetry breaking mechanism. Moreover, it is relevant to emphasize again that by construction the model is renormalizable due to the geometrical properties of the tensorial fields. Finally, one can note that mixing terms between the tensorial terms and the gauge curvature take into account the values of a scalar field and can be useful to study topological properties of this action, but this is a very extended task and certainly demands another work.

\section{CONCLUSION}

In this work, we present a possible extension of the main idea presented in Ref. [32] that is to localize a non-Abelian gauge-invariant operator in order to obtain gauge-invariant mass term. The method uses a symmetry breaking mechanism and obtain the same mass for all components of the gauge field. This can be useful in order to study the operator $A_{\min }^{2}$ that is quite important in many aspects of confinement [34-39]. We present all the necessary equations that are compatible to the quantum action principle that can be used to prove the renormalizability of the model. We have obtained the important property that mass terms in the localizing fields are blocked by the geometrical properties of these fields while maintaining the localizing property. We point out that this action possesses a small number of parameters, a feature that is useful for higher order computations.

The possibility of having at our disposal a true local and renormalizable action might provide us with a consistent framework for a future investigation of the possible implications of nonlocal gauge-invariant operators of ultraviolet dimension two.

\section{ACKNOWLEDGMENTS}

This study was financed in part by the Coordenação de Aperfeiçoamento de Pessoal de Nível Superior-Brasil (Capes)-Finance Code 001. The Conselho Nacional de Desenvolvimento Científico e Tecnológico (CNPq-Brazil) and the SR2-UERJ are gratefully acknowledged for financial support. M. A. L. C. is a level PQ-2 researcher under the program Produtividade em Pesquisa-CNPq, 302040/ 2017-0.
[1] J. M. Cornwall, Phys. Rev. D 26, 1453 (1982).

[2] J. Greensite and M. B. Halpern, Nucl. Phys. B271, 379 (1986).

[3] M. Stingl, Phys. Rev. D 34, 3863 (1986); 36, 651(E) (1987).

[4] M. J. Lavelle and M. Schaden, Phys. Lett. B 208, 297 (1988).

[5] F. V. Gubarev and V. I. Zakharov, Phys. Lett. B 501, 28 (2001).

[6] F. V. Gubarev, L. Stodolsky, and V. I. Zakharov, Phys. Rev. Lett. 86, 2220 (2001).

[7] H. Verschelde, K. Knecht, K. Van Acoleyen, and M. Vanderkelen, Phys. Lett. B 516, 307 (2001).

[8] K. I. Kondo, Phys. Lett. B 514, 335 (2001).

[9] K. I. Kondo, T. Murakami, T. Shinohara, and T. Imai, Phys. Rev. D 65, 085034 (2002).
[10] D. Dudal, H. Verschelde, R. E. Browne, and J. A. Gracey, Phys. Lett. B 562, 87 (2003).

[11] R. E. Browne and J. A. Gracey, J. High Energy Phys. 11 (2003) 029.

[12] D. Dudal, H. Verschelde, J. A. Gracey, V. E. R. Lemes, M. S. Sarandy, R. F. Sobreiro, and S. P. Sorella, J. High Energy Phys. 01 (2004) 044.

[13] D. Dudal, J. A. Gracey, V. E. R. Lemes, M. S. Sarandy, R. F. Sobreiro, S. P. Sorella, and H. Verschelde, Phys. Rev. D 70, 114038 (2004).

[14] R. E. Browne and J. A. Gracey, Phys. Lett. B 597, 368 (2004).

[15] J. A. Gracey, Eur. Phys. J. C 39, 61 (2005).

[16] X.d. Li and C.M. Shakin, Phys. Rev. D 71, 074007 (2005). 
[17] P. Boucaud, A. Le Yaouanc, J. P. Leroy, J. Micheli, O. Pene, and J. Rodriguez-Quintero, Phys. Rev. D 63, 114003 (2001).

[18] P. Boucaud, J. P. Leroy, A. Le Yaouanc, J. Micheli, O. Pene, F. De Soto, A. Donini, H. Moutare, and J. RodriguezQuintero, Phys. Rev. D 66, 034504 (2002).

[19] P. Boucaud, F. de Soto, J. P. Leroy, A. Le Yaouanc, J. Micheli, H. Moutarde, O. Pene, and J. Rodriguez-Quintero, Phys. Rev. D 74. 034505 (2006).

[20] E. Ruiz Arriola, P. O. Bowman, and W. Broniowski, Phys. Rev. D 70, 097505 (2004).

[21] T. Suzuki, K. Ishiguro, Y. Mori, and T. Sekido, Phys. Rev. Lett. 94, 132001 (2005).

[22] F. V. Gubarev and S. M. Morozov, Phys. Rev. D 71, 114514 (2005).

[23] S. Furui and H. Nakajima, Few Body Syst. 40, 101 (2006).

[24] P. Boucaud, J. P. Leroy, A. Le Yaouanc, A. Y. Lokhov, J. Micheli, O. Pene, J. Rodriguez-Quintero, and C. Roiesnel, J. High Energy Phys. 01 (2006) 037.

[25] M. N. Chernodub, K. Ishiguro, Y. Mori, Y. Nakamura, M. I. Polikarpov, T. Sekido, T. Suzuki, and V. I. Zakharov, Phys. Rev. D 72, 074505 (2005).

[26] D. Dudal, H. Verschelde, V. E. R. Lemes, M. S. Sarandy, S. P. Sorella, and M. Picariello, Ann. Phys. (Amsterdam) 308, 62 (2003).

[27] D. Dudal, H. Verschelde, V. E. R. Lemes, M. S. Sarandy, R. F. Sobreiro, S. P. Sorella, and J. A. Gracey, Phys. Lett. B 574, 325 (2003).
[28] D. Dudal, H. Verschelde, V. E. R. Lemes, M. S. Sarandy, R. F. Sobreiro, S. P. Sorella, M. Picariello, and J. A. Gracey, Phys. Lett. B 569, 57 (2003) [arXiv:hep-th/0306116].

[29] R. Delbourgo and G. Thompson, Phys. Rev. Lett. 57, 2610 (1986).

[30] R. Delbourgo, S. Twisk, and G. Thompson, Int. J. Mod. Phys. A 03, 435 (1988).

[31] H. Ruegg and M. Ruiz-Altaba, Int. J. Mod. Phys. A 19, 3265 (2004).

[32] M. A. L. Capri, D. Dudal, J. A. Gracey, V. E. R. Lemes, R. F. Sobreiro, S. P. Sorella, and H. Verschelde, Phys. Rev. D 72, 105016 (2005).

[33] O. Piguet and S. P. Sorella, Lect. Notes Phys. Monographs 28, 1 (1995).

[34] M. A. Semenov-Tyan-Shanskii and V. A. Franke, Zapiski Nauchnykh Seminarov Leningradskogo Otdeleniya Matematicheskogo Instituta im. V.A. Steklov AN SSSR 120, 159 (1982) [J. Sov. Math. 34, 1999 (1986)].

[35] D. Zwanziger, Nucl. Phys. B345, 461 (1990).

[36] G. Dell'Antonio and D. Zwanziger, Nucl. Phys. B326, 333 (1989).

[37] G. Dell'Antonio and D. Zwanziger, Commun. Math. Phys. 138, 291 (1991).

[38] D. Dudal, H. Verschelde, V. E. R. Lemes, M. S. Sarandy, S. P. Sorella, and M. Picariello, Ann. Phys. (Amsterdam) 308, 62 (2003).

[39] P. van Baal, Nucl. Phys. B369, 259 (1992). 